\title{
Decreased HECTD1 mRNA expression is associated with poor prognosis and enhanced mitochondrial cellular respiratory function in breast cancer
}

\section{Yasuaki Uemoto}

Nagoya City University Graduate School of Medical Sciences

\section{Eriko Katsuta}

Roswell Park Comprehensive Cancer Center

\section{Naoto Kondo}

Nagoya City University Graduate School of Medical Sciences

\section{Yumi Wanifuchi-Endo}

Nagoya City University Graduate School of Medical Sciences

\section{Takashi Fujita}

Nagoya City University Graduate School of Medical Sciences

\section{Tomoko Asano}

Nagoya City University Graduate School of Medical Sciences

\section{Tomoka Hisada}

Nagoya City University Graduate School of Medical Sciences

\section{Mitsuo Terada}

Nagoya City University Graduate School of Medical Sciences

\section{Akiko Kato}

Nagoya City University Graduate School of Medical Sciences

\section{Katsuhiro Okuda}

Nagoya City University Graduate School of Medical Sciences Hiroshi Sugiura

Nagoya City University Graduate School of Medical Sciences Hiroyuki Kato

Nagoya City University Graduate School of Medical Sciences

\section{Satoshi Osaga}

Nagoya City University Hospital

\section{Satoru Takahashi}

Nagoya City University Graduate School of Medical Sciences

Tatsuya Toyama ( $\nabla$ t.toyama@med.nagoya-cu.ac.jp )

Nagoya City University Graduate School of Medical Sciences 


\section{Research Article}

Keywords: HECTD1 mRNA expression, mitochondrial cellular respiratory function , breast cancer Posted Date: May 13th, 2021

DOI: https://doi.org/10.21203/rs.3.rs-514566/v1

License: (c) (i) This work is licensed under a Creative Commons Attribution 4.0 International License. Read Full License 


\section{Abstract}

HECT domain E3 ubiquitin ligase 1 (HECTD1) has been reported to be a negative regulator of epithelialmesenchymal transition and to decrease breast cancer invasion and metastasis. However, the clinical significance and detailed role of HECTD1 in breast cancer remain elusive. We investigated the role of HECTD1 in two large breast cancer cohorts using mRNA and protein expression, and bioinformatics. We examined the prognostic significance of HECTD1 by multivariate analysis. HECTD1 mRNA expression (HECTD1 expression) was lower in breast cancer compared with adjacent normal tissues. HECTD1 expression levels also differed among breast cancer subtypes. Decreased HECTD1 expression was significantly associated with aggressive tumour characteristics, including large tumour size and high histological grade. HECTD1 expression was inversely associated with mitochondrial cellular respiratory function and reactive oxygen species in breast cancer tissues. Multivariate analysis identified low HECTD1 mRNA expression level as an independent risk factor for disease-free $(P=0.009)$ and overall ( $P$ $=0.046)$ survival among breast cancer patients. There was no association of HECTD1 protein expression with mRNA expression and prognosis. HECTD1 mRNA expression is a candidate prognostic biomarker in breast cancer. The poor prognosis of patients with low HECTD1 mRNA expression may be associated with increased mitochondrial respiratory function.

\section{Introduction}

Breast cancer is the most prevalent malignancy in women and its incidence is still increasing ${ }^{1}$. Although breast cancer mortality has decreased as a result of improvements in systemic therapy ${ }^{2,3}$, breast cancer remains the leading cause of cancer-related death in women worldwide. Further research is thus needed to understand the molecular mechanisms of breast cancer and to improve the prognosis of breast cancer patients.

Ubiquitination is an important post-translational enzymatic protein modification that is mediated by a three-enzyme cascade (E1, E2, and E3) ${ }^{4}$. Ubiquitinated proteins are subsequently degraded by the proteasome ${ }^{5,6}$. HECT domain E3 ubiquitin ligase 1 (HECTD1) negatively regulates the functions of specific target proteins through mediating ubiquitination, leading to subsequent protein degradation. HECTD1 was previously shown to play crucial roles in the negative regulation of cell ${ }^{7-10}$ migration. A recent report demonstrated that HECTD1 negatively regulates epithelial-mesenchymal transition (EMT), resulting in decreased invasion and metastasis in breast cancer ${ }^{11}$. However, the clinical significance and detailed roles of HECTD1 in breast cancer are still elusive.

Mitochondria are the major producers of ATP through oxidative phosphorylation (OXPHOS) in normal cells. OXPHOS, which produces ATP using oxygen via the respiratory chain complex and generates reactive oxygen species (ROS), is one of the typical processes of mitochondrial cellular respiration ${ }^{12}$. Although glycolysis is dominant in cancer metabolism, mitochondria also play a critical role in the development of some types of cancer ${ }^{13}$. For example, one study showed that mitochondrial respiration 
contributed more to ATP production compared with glycolysis in ovarian and lung cancers ${ }^{14}$. Enhanced mitochondrial respiration was also reported to be associated with cancer cell invasion and metastasis ${ }^{15}$. ROS accumulation was also shown to promote tumorigenesis and cancer progression by mediating genomic instability ${ }^{16}$. Interestingly, mitochondria are reported to be targeted for degradation through ubiquitination by an E3 ubiquitin ligase ${ }^{17}$.

Given that HECTD1 regulates protein functions via its ubiquitination activity as an E3 ubiquitin ligase, we hypothesized that HECTD1 suppresses mitochondrial respiration in breast cancer and is thus negatively associated with a poor prognosis. To this end, we investigated the possible roles of HECTD1 in relation to mitochondrial respiration and clinicopathological factors, including prognosis, in two large cohorts of breast cancer patients.

\section{Results}

\section{HECTD1 mRNA was downregulated in breast cancer tissues}

We first investigated if HECTD1 mRNA expression levels are altered in breast cancer tissues by analyzing 114 matched tumor and adjacent normal tissues in TCGA cohort. We found that HECTD1 mRNA expression levels were significantly lower in the breast tumor tissues compared with the adjacent normal tissues $(P<0.001)$ (Fig. 1).

\section{HECTD1 expression differed among breast cancer subtypes}

We examined if HECTD1 mRNA expression levels are different among breast cancer subtypes in the NCU cohort. HECTD1 mRNA expression levels were significantly lower in ER-negative tumors $(P<0.001)$, PgRnegative tumors $(P<0.001)$, and HER2-positive tumors $(P<0.001)$ compared with the respective counterparts (Fig. 2). We further stratified the patients into four subtypes based on IHC findings: luminal (ER-positive and/or PgR-positive, HER2-negative), luminal-HER2 (ER-positive and/or PgR-positive, HER2positive), HER2 (ER-negative, PgR-negative, HER2-positive), and triple-negative (ER-negative, PgR-negative, HER2-negative). HECTD1 mRNA expression levels were significantly lower in the HER2 and triple-negative subtypes compared with the luminal subtype ( $P=0.001$ and $P<0.001$, respectively) (Fig. 2$)$.

\section{HECTD1 mRNA level was negatively associated with aggressive tumor characteristics}

Based on the downregulation of HECTD1 mRNA in tumor tissues (Fig. 1) and the previous reports showing that HECTD1 suppressed EMT ${ }^{10,11}$, we investigated the association between HECTD1 mRNA expression and tumor aggressiveness in the NCU cohort. HECTD1 mRNA expression was significantly lower in larger tumors ( $P=0.009$ ) and higher-grade tumors (grade 3 vs. grade $1, \mathrm{P}<0.001$; grade 3 vs. grade 2, $P<0.001$; grade 2 vs. grade 1, $P=0.042$ ) (Fig. 3). However, HECTD1 mRNA expression was not associated with lymph node metastasis. 
We also investigated the associations between HECTD1 mRNA expression levels and other patient demographics in the NCU cohort. High HECTD1 mRNA expression was associated with a higher proportion of invasive lobular carcinomas compared with tumors with low HECTD1 expression $(P=0.009)$ (Supplementary Table S1). However, because of the small number of lobular carcinomas, the results could not be determined to be statistically significant. There was no significant association between HECTD1 mRNA expression and other examined factors, including age, sex, or menopausal status.

\section{HECTD1 was inversely associated with genes involved in mitochondrial cellular respiratory function}

HECTD1 was shown to negatively regulate EMT (Duhamel et al. 2018). We therefore investigated the relationship between HECTD1 mRNA expression and EMT using TCGA cohorts. In contrast to the previous report (Duhamel et al. 2018), GSEA revealed no correlation between HECTD1 mRNA expression and the EMT gene set (Fig. 4a). In contrast, we found that the mitochondrial gene set, mitochondrion $(P=0.009)$, and especially the respiratory-related gene sets for OXPHOS $(P=0.011)$, the respiratory chain complex $(P=0.006)$, and the ROS pathway $(P=0.009)$ were inversely correlated with HECTD1 mRNA expression in TCGA cohort (Fig. 4b). These findings thus indicated that HECTD1 might be directly or indirectly involved in the negative regulation of mitochondrial cellular respiratory function in breast cancer.

\section{Low HECTD1 mRNA expression was associated with poor prognosis in breast cancer}

We analyzed the impact of HECTD1 mRNA expression on the survival of breast cancer patients. Patients with HECTD1 mRNA low-expressing tumors had significantly shorter DFS and OS among all breast cancer patients ( $P=0.013$ and $P=0.038$, respectively) in the NCU cohort (Fig. 5a). Univariate analyses showed that larger tumor size (DFS, $P=0.032$; OS, $P=0.002$ ), lymph node metastasis (DFS, $P<0.001$; OS, $P<0.001$ ), higher histological grade (DFS, $P=0.003$; OS, $P=0.002$ ), ER-negativity (DFS, $P=0.004$; OS, $P=0.005$ ), HER2-positivity (DFS, $P=0.015$; OS, $P=0.011$ ), and low HECTD1 mRNA expression level (DFS, $P=0.014$; OS, $P=0.039$ ) were all significantly associated with shorter DFS and OS (Table 1). Multivariate analyses identified low HECTD1 mRNA expression level as an independent factor associated with lower DFS (hazard ratio (HR): 1.54, 95\% confidence interval (Cl): 1.11-2.13, $P=0.009$ ), together with lymph node metastasis $(P<0.001)$ and ER status $(P=0.009)$ (Table 1). Low HECTD1 mRNA expression was also an independent prognostic factor for poor OS (HR: 1.50,95\% Cl: 1.01-2.24, $P=0.046$ ), together with lymph node metastasis $(P<0.001)$ and ER status $(P=0.037)$ (Table 1$)$.

Given that HECTD1 mRNA expression levels differed according to subtype, we further investigated the impact of HECTD1 mRNA expression on patient survival in each subtype. There was no significant difference in either DFS or OS between the HECTD1 high- and low-expressing tumors in ER-positive (DFS, $P=0.273$; OS, $P=0.388$ ) (Fig. 5b) and HER2-positive patients (DFS, $P=0.617$; OS, $P=0.972$ ) (Supplementary Fig. S1 online). However, low HECTD1 expression tended to be associated with worse prognosis in ERnegative (DFS, $P=0.079$; OS, $P=0.139$ ) (Fig. 5 c) and triple-negative patients (DFS, $P=0.123$; OS, $P=0.106$ ) (Supplementary Fig. S1 online). 


\section{Lack of association of HECTD1 protein expression with mRNA expression or patient survival in breast cancer}

We investigated if HECTD1 mRNA expression was correlated with its protein expression levels in breast cancer. Representative images of HECTD1 protein expression detected by IHC are shown in Fig. 6a. There was no significant correlation between HECTD1 mRNA expression levels and HECTD1 H-score in the NCU cohort ( $\mathrm{r}=-0.089, P=0.134$ ) (Fig. 6b) or protein expression by mass spectrometry in TCGA cohort $(\mathrm{r}=0.205$, $P=0.079$ ) (Fig. 6c). In addition, there was no significant difference in DFS or OS between patients with HECTD1 high- and low-expressing tumors in the NCU cohort ( $P=0.698$ and $P=0.819$, respectively) (Fig. $6 d)$.

\section{Discussion}

The results of this study revealed that HECTD1 mRNA expression levels were lower in breast cancer tissues compared with normal adjacent tissues. HECTD1 mRNA expression levels were different among breast cancer subtypes. We demonstrated that low HECTD1 mRNA expression was associated with aggressive tumor characteristics, such as large tumor size and high histological grade, and was an independent poor prognostic factor in breast cancer patients. We identified an inverse correlation between HECTD1 mRNA expression and genes involved in mitochondrial cellular respiratory function as a possible mechanism for these relationships.

Low protein expression of HECTD1 in breast cancer as determined by $\mathrm{IHC}$ has previously been reported ${ }^{11}$; however, HECTD1 mRNA levels in cancer have not been reported to date. The current results provide the first evidence for the downregulation of HECTD1 mRNA expression in breast tumors compared with adjacent normal tissues. Depletion of HECTD1 has been reported to lead to increased breast cancer cell proliferation and invasion ${ }^{8,11}$. These studies support our findings that low mRNA expression of HECTD1 was associated with aggressive tumor characteristics, such as larger size and higher grade.

Breast cancer subtypes, classified by IHC analyses of ER, PgR, and HER2, are known to have different biological characteristics and prognoses. We found that HECTD1 mRNA expression varied among the breast cancer subtypes, with low expression in ER-negative, PgR-negative and HER2-positive tumors compared with the respective counterparts. These findings might be consistent with the fact that ERnegative, PgR-negative and HER2-positive breast cancers tend to have more aggressive features and poorer clinical outcomes ${ }^{18,19}$. Furthermore, the impact of HECTD1 mRNA expression on patient prognosis also differed by subtypes, with low HECTD1 mRNA expression levels tending to be associated with a poorer prognosis in ER-negative and triple-negative breast cancer patients but not ER-positive and HER2positive breast cancer patients. These findings suggest that HECTD1 plays different roles in different breast cancer subtypes.

Although previous reports indicated that HECTD1 negatively regulates $\mathrm{EMT}^{11}$, no association was shown between HECTD1 mRNA expression and EMT in the current study results. We found a lack of association 
between HECTD1 mRNA expression and lymph node metastasis as well as EMT-related genes by GSEA. These results imply that HECTD1 may have a different role in breast cancer other than previously reported.

OXPHOS, one of the typical processes of mitochondrial respiration, efficiently produces ATP via the respiratory chain complex using oxygen and generating ROS ${ }^{12}$. Enhanced mitochondrial OXPHOS in breast cancer was reported to be associated with cancer cell motility, distant metastasis, and poor patient prognosis through high ATP production ${ }^{20}$. The increase in ROS associated with enhancement of OXPHOS was also reported to promote cancer cell proliferation via multiple signaling pathways ${ }^{21,22}$. In the current study, we demonstrated that mitochondrial respiratory functions, such as OXPHOS, the respiratory chain complex, and ROS, were inversely correlated with HECTD1 mRNA expression. These results imply that HECTD1 mRNA expression may be involved in the negative regulation of mitochondrial respiratory function, resulting in reduced cancer aggressiveness and better survival.

The current study found no correlation between HECTD1 mRNA levels and protein levels measured by IHC in the NCU cohort or protein levels measured by mass spectrometry in the TCGA cohort. Although the mechanism that regulates HECTD1 protein expression has not yet been fully elucidated, it might involve translational control ${ }^{23,24}$ and post-transcriptional modification ${ }^{25,26}$. Furthermore, in this study, there was an association between HECTD1 mRNA expression and breast cancer prognosis and mitochondrial respiratory function, but no association between HECTD1 protein expression and breast cancer prognosis. These findings imply that HECTD1 is associated with breast cancer prognosis by not only suppressing EMT via protein function as previously reported ${ }^{11}$, but also by suppressing mitochondrial respiratory function via mRNA expression as suggested in this study. Further studies are required to investigate the mechanism regulating mitochondrial respiratory function via HECTD1 mRNA expression.

This study has several limitations. First, this was a retrospective analysis at a single institution using archived materials. Second, the IHC methodology for HECTD1 protein evaluation has not yet been well established. Third, the role of HECTD1 in breast cancer was only investigated using in silico analysis, and further in vitro and in vivo experimental approaches are needed to verify the role of HECTD1 in breast cancer.

In summary, we identified low expression of HECTD1 mRNA as an independent poor prognostic factor in breast cancer and that HECTD1 mRNA expression was inversely correlated with genes involved in mitochondrial cellular respiratory function in breast cancer. These findings warrant further studies to elucidate the role of HECTD1 in breast cancer.

\section{Methods}

\section{Patients and samples}


A total 625 consecutive invasive breast cancer tissue samples from the Nagoya City University Hospital archive (collected between 1992 and 2008) were included in this study (the NCU cohort). Among the 625 samples, 340 had only mRNA expression data available; the other 285 cases, which were collected between 2000 and 2008, had both mRNA and protein expression data available. Information of patient clinical parameters was collected from the clinical records. Histological tumor grade was estimated according to the Bloom and Richardson method proposed by Elston and Ellis (Elston and Ellis 1991). Disease-free survival (DFS) was defined as the interval from the date of curative resection to the earliest occurrence of locoregional recurrence, distant metastasis, or death from any cause. Overall survival (OS) was defined as the interval from the date of curative resection to death from any cause. The median follow-up periods in the mRNA and protein expression cohorts were 10.2 years (range, $0.01-17.9$ years) and 9.8 years (range, $0.07-17.9$ years), respectively. The study protocol (70-00-0166) was approved by the Institutional Review Board of Nagoya City University Graduate School of Medical Sciences and conformed to the guidelines of the Declaration of Helsinki. Written informed consent for comprehensive research use was obtained from all patients before surgery.

\section{The Cancer Genome Atlas (TCGA) data acquisition}

The data of gene expression from RNA sequencing and protein expression from mass spectrometry of breast cancer patients in TCGA provisional cohort were downloaded through cBioPortal (www.cbioportal.org) ${ }^{27,28}$ and the UCSC Genome Browser (http://genome.ucsc.edu/). Of the 1093 TCGA breast cancer patients with gene expression data from RNA sequencing, 74 patients also had protein expression data from mass spectrometry and 114 patients had gene expression data of matched adjacent normal tissues.

\section{Gene set enrichment analysis (GSEA)}

GSEA was performed using data from TCGA cohort to analyze correlations with HECTD1 mRNA expression. HECTD1 mRNA expression-related gene sets were identified within the 50 hallmark gene sets $^{29}$, the Molecular Signature Database curated collection (c2), and Gene Ontology gene sets (c5).

\section{RNA extraction and quantitative reverse transcription polymerase chain reaction (qRT-PCR)}

Breast cancer tissue samples were snap-frozen in liquid nitrogen immediately after resection and stored at $-80^{\circ} \mathrm{C}$ until RNA extraction. Total RNA was isolated using the RNeasy Mini Kit (Qiagen, Tokyo, Japan) according to the manufacturer's protocol. The quantity of RNA extracted from breast cancer tissues was evaluated using a DS-11 Spectrophotometer (DeNovix, Wilmington, DE, USA). Reverse transcription was performed using a High Capacity cDNA Reverse Transcription Kit (Thermo Fisher Scientific, Waltham, MA, USA) according to the manufacturer's protocol. qRT-PCR was carried out on a Step One Plus ${ }^{\text {TM }}$ Real-time PCR system (Thermo Fisher Scientific). Reactions were multiplexed using the following assays: HECTD1 (FAM; Thermo Fisher Scientific) and GAPDH (VIC; Life Technologies, Waltham, MA, USA) using Fast Advanced Master Mix (Applied Biosystems, Waltham, MA, USA). The results were converted into gene expression levels using a standard curve. Target gene expression was normalized relative to levels of the 
GAPDH gene, as described previously ${ }^{30,31}$. We determined the cut-off level for HECTD1 mRNA expression level as the median value.

\section{Immunohistochemistry (IHC)}

IHC analyses for estrogen receptor (ER), progesterone receptor (PgR), and human epidermal growth factor 2 (HER2) were carried out as described elsewhere ${ }^{31,32}$. For HECTD1 staining, a tissue microarray was constructed using 2-mm diameter tissue samples. After deparaffinization, antigens were retrieved by heating sections at $99-100^{\circ} \mathrm{C}$ in $0.01 \mathrm{M}$ citrate buffer $\mathrm{pH} 6$ for $20 \mathrm{~min}$. Endogenous peroxidase activity was inhibited by incubation in $3 \% \mathrm{H}_{2} \mathrm{O}_{2}$ for $10 \mathrm{~min}$. The slides were then incubated in Protein Block solution (DS Pharma Biomedical Co., Osaka, Japan) for $10 \mathrm{~min}$ to minimize nonspecific staining, followed by incubation with rabbit polyclonal anti-human HECTD1 antibody (LifeSpan BioSciences, Seattle, WA, USA) at a 1:100 dilution overnight at $4^{\circ} \mathrm{C}$. HECTD1 was then detected using the EnVision system, peroxidase (Dako, Santa Clara, CA, USA), and diaminobenzidene detection (Merck KGaA, Darmstadt, Germany). HECTD1 protein expression level was scored by assessing the entire slide using the Aperio ImageScope system (Leica Biosystems, Vista, CA, USA). Cytoplasmic staining intensity $(0,1+$, $2+$, or $3+)$ was determined for each cancer cell, and an $\mathrm{H}$-score was assigned using the formula [1 $\times(\%$ cells $1+)+2 \times(\%$ cells $2+)+3 \times(\%$ cells $3+)]^{33,34}$. We determined the cut-off level for HECTD $1 \mathrm{H}$-score as the median.

\section{Statistical analyses}

HECTD1 mRNA expression levels were compared using Mann-Whitney and pairwise Mann-Whitney tests with Bonferroni's adjustment. Spearman's rank correlation coefficient ( $r$ ) was used to analyze correlations between HECTD1 mRNA expression levels and with HECTD1 $\mathrm{H}$-score and protein level. Associations between clinicopathological factors and HECTD1 mRNA expression levels were assessed by Student's $t-, \chi^{2}$, and Fisher's exact probability tests. Survival analyses were performed using KaplanMeier curves with the log-rank test. Univariate and multivariate analyses were carried out using Cox proportional hazards regression models, and a total of 69 missing data points were estimated using multiple imputation. A $P$-value $<0.05$ was considered significant. All statistical analyses were performed with R software version 4.0.0 (https://www.R-project.org/) and Bioconductor version 3.11 (http://bioconductor.org/). GSEA was performed using the Java GSEA implementation version 4.0.3 and MSigDB version 6.0 .

\section{Declarations}

\section{Data availability}

The NCU datasets analyzed during the current study are available from the corresponding author on reasonable request.

\section{Ethics declarations}


This study protocol (70-00-0166) was approved by the Institutional Review Board of Nagoya City University Graduate School of Medical sciences. All tissue samples were provided by a biobank that is maintained by the Department of Breast Surgery, Nagoya City University Graduate School of Medical Sciences, which conforms to the guidelines of the guidelines of the Declaration of Helsinki. Written informed consent for the comprehensive research use of clinical samples was obtained from all patients involved in this study.

\section{Acknowledgements}

This research was supported in part by a Grant-in-Aid for Scientific Research from the Japanese Ministry of Education, Culture, Science, and Technology. We are grateful to the patients in this study. We thank Mrs. Makino for her excellent technical assistance. We also thank Susan Furness, PhD, from Edanz Group (https://en-author-services.edanz.com/ac) for editing a draft of this manuscript.

\section{Author contributions}

YU, EK, and TT conceived the project, designed experiments, analysed and interpreted data. YU and EK performed experiments. YU, EK, and SO performed statistical analyses. YU wrote the paper. YU, EK, and TT revised the paper. YU, TT, NK, YW-E, TF, HS, TA, TH, MT, AK, HK, and ST collected the data. All authors critically reviewed the paper and had final approval of it.

\section{Additional Information}

Competing interests: The authors declare no competing interests.

\section{References}

1. Sharma, R. Breast cancer incidence, mortality and mortality-to-incidence ratio (MIR) are associated with human development, 1990-2016: evidence from Global Burden of Disease Study 2016. Breast cancer (Tokyo, Japan) 26, 428-445 (2019).

2. Kohler, B. A. et al. Annual Report to the Nation on the Status of Cancer, 1975-2011, Featuring Incidence of Breast Cancer Subtypes by Race/Ethnicity, Poverty, and State. Journal of the National Cancer Institute 107, djv048 (2015).

3. de Gelder, R., Heijnsdijk, E. A. M., Fracheboud, J., Draisma, G. \& de Koning, H. J. The effects of population-based mammography screening starting between age 40 and 50 in the presence of adjuvant systemic therapy. International journal of cancer 137, 165-172 (2015).

4. Rotin, D. \& Kumar, S. Physiological functions of the HECT family of ubiquitin ligases. Nature Reviews Molecular Cell Biology 10, 398-409 (2009).

5. Pickart, C. M. \& Eddins, M. J. Ubiquitin: Structures, functions, mechanisms. Biochimica et Biophysica Acta - Molecular Cell Research 1695, 55-72 (2004). 
6. Metzger, M. B., Hristova, V. A. \& Weissman, A. M. HECT and RING finger families of E3 ubiquitin ligases at a glance. Journal of Cell Science 125, 531-537 (2012).

7. Sarkar, A. A. \& Zohn, I. E. Hectd1 regulates intracellular localization and secretion of Hsp90 to control cellular behavior of the cranial mesenchyme. Journal of Cell Biology 196, 789-800 (2012).

8. Li, X. et al. Ubiquitylation of phosphatidylinositol 4-phosphate 5-kinase type I y by HECTD1 regulates focal adhesion dynamics and cell migration. Journal of Cell Science 126, 2617-2628 (2013).

9. Tran, H. et al. HectDI E3 ligase modifies adenomatous polyposis coli (APC) with polyubiquitin to promote the APC-axin interaction. Journal of Biological Chemistry 288, 3753-3767 (2013).

10. Wang, X., de Geyter, C., Jia, Z., Peng, Y. \& Zhang, H. HECTD1 regulates the expression of SNAIL: Implications for epithelial-mesenchymal transition. International Journal of Oncology 56, 11861198 (2020).

11. Duhamel, S. et al. The E3 Ubiquitin Ligase HectD1 Suppresses EMT and Metastasis by Targeting the +TIP ACF7 for Degradation. Cell Reports 22, 1016-1030 (2018).

12. Zong, W.-X., Rabinowitz, J. D. \& White, E. Mitochondria and Cancer. Molecular cell 61, 667-676 (2016).

13. Jose, C., Bellance, N. \& Rossignol, R. Choosing between glycolysis and oxidative phosphorylation: a tumor's dilemma? Biochimica et biophysica acta 1807, 552-561 (2011).

14. Zu, X. L. \& Guppy, M. Cancer metabolism: Facts, fantasy, and fiction. Biochemical and Biophysical Research Communications 313, 459-465 (2004).

15. Rivadeneira, D. B. et al. Survivin promotes oxidative phosphorylation, subcellular mitochondrial repositioning, and tumor cell invasion. Science Signaling 8, 1-13 (2015).

16. Tanaka, S. et al. Increased hepatic oxidative DNA damage in patients with nonalcoholic steatohepatitis who develop hepatocellular carcinoma. Journal of gastroenterology $48,1249-1258$ (2013).

17. Riley, B. E. et al. Structure and function of Parkin E3 ubiquitin ligase reveals aspects of RING and HECT ligases. Nature Communications 4, (2013).

18. Fan, C. et al. Concordance among gene-expression-based predictors for breast cancer. New England Journal of Medicine 355, 560-569 (2006).

19. Gyorffy, B. et al. Multigene prognostic tests in breast cancer: Past, present, future. Breast Cancer Research 17, 1-7 (2015).

20. Lebleu, V. S. et al. PGC-1a mediates mitochondrial biogenesis and oxidative phosphorylation in cancer cells to promote metastasis. Nature Cell Biology 16, 992-1003 (2014).

21. Shadel, G. S. \& Horvath, T. L. Mitochondrial ROS Signaling in Organismal Homeostasis. Cell 163, 560-569 (2015).

22. Deng, W. et al. MICAL1 facilitates breast cancer cell proliferation via ROS-sensitive ERK/cyclin D pathway. Journal of cellular and molecular medicine 22, 3108-3118 (2018). 
23. Gebauer, F. \& Hentze, M. W. Molecular mechanisms of translational control. Nature Reviews Molecular Cell Biology 5, 827-835 (2004).

24. Sonenberg, N. \& Hinnebusch, A. G. Regulation of Translation Initiation in Eukaryotes: Mechanisms and Biological Targets. Cell 136, 731-745 (2009).

25. Venne, A. S., Kollipara, L. \& Zahedi, R. P. The next level of complexity: crosstalk of posttranslational modifications. Proteomics 14, 513-524 (2014).

26. Liu, Y., Beyer, A. \& Aebersold, R. On the Dependency of Cellular Protein Levels on mRNA Abundance. Cell 165, 535-550 (2016).

27. Cerami, E. et al. The cBio cancer genomics portal: an open platform for exploring multidimensional cancer genomics data. Cancer discovery 2, 401-404 (2012).

28. Wu, P. et al. Integration and Analysis of CPTAC Proteomics Data in the Context of Cancer Genomics in the cBioPortal. Molecular \& cellular proteomics: MCP 18, 1893-1898 (2019).

29. Liberzon, A. et al. The Molecular Signatures Database (MSigDB) hallmark gene set collection. Cell systems 1, 417-425 (2015).

30. Čikoš, Š., Bukovská, A. \& Koppel, J. Relative quantification of mRNA: Comparison of methods currently used for real-time PCR data analysis. BMC Molecular Biology 8, 1-14 (2007).

31. Nishimoto, M. et al. Prognostic impact of TP53INP1 gene expression in estrogen receptor a-positive breast cancer patients. Japanese journal of clinical oncology 49, 567-575 (2019).

32. Harvey, J. M., Clark, G. M., Osborne, C. K. \& Allred, D. C. Estrogen receptor status by immunohistochemistry is superior to the ligand-binding assay for predicting response to adjuvant endocrine therapy in breast cancer. Journal of clinical oncology: official journal of the American Society of Clinical Oncology 17, 1474-1481 (1999).

33. Hirsch, F. R. et al. Epidermal growth factor receptor in non-small-cell lung carcinomas: Correlation between gene copy number and protein expression and impact on prognosis. Journal of Clinical Oncology 21, 3798-3807 (2003).

34. John, T., Liu, G. \& Tsao, M. S. Overview of molecular testing in non-small-cell lung cancer: Mutational analysis, gene copy number, protein expression and other biomarkers of EGFR for the prediction of response to tyrosine kinase inhibitors. Oncogene 28, 14-23 (2009).

\section{Table}




\begin{tabular}{|c|c|c|c|c|c|c|}
\hline \multirow{3}{*}{$\begin{array}{l}\mathrm{n} \\
(\%)\end{array}$} & \multicolumn{3}{|c|}{ Disease-free survival } & \multicolumn{3}{|c|}{ Overall survival } \\
\hline & \multirow{2}{*}{$\begin{array}{l}\text { Univariate } \\
P \text { value }\end{array}$} & \multicolumn{2}{|c|}{ Multivariate } & \multirow{2}{*}{$\begin{array}{l}\text { Univariate } \\
P \text { value }\end{array}$} & \multicolumn{2}{|c|}{ Multivariate } \\
\hline & & $P$ value & $\begin{array}{l}\text { HR }(95 \% \\
\text { CI) }\end{array}$ & & $P$ value & $\begin{array}{l}\text { HR }(95 \% \\
\text { CI })\end{array}$ \\
\hline 625 & 0.233 & 0.194 & $\begin{array}{l}1.01(0.99- \\
1.02)\end{array}$ & 0.130 & 0.105 & $\begin{array}{l}1.01(0.99- \\
1.03)\end{array}$ \\
\hline 624 & & & & & & \\
\hline $\begin{array}{l}241 \\
(39) \\
383 \\
(61) \\
597\end{array}$ & 0.032 & 0.452 & $\begin{array}{l}1 \\
\text { (Reference) } \\
1.14(0.81- \\
1.59)\end{array}$ & 0.002 & 0.062 & $\begin{array}{l}1 \\
\text { (Reference) } \\
1.54(0.98- \\
2.41)\end{array}$ \\
\hline $\begin{array}{l}340 \\
(60) \\
257 \\
(40) \\
600\end{array}$ & $<0.001$ & $<0.001^{*}$ & $\begin{array}{l}1 \\
\text { (Reference) } \\
4.18(2.97- \\
5.88)\end{array}$ & $<0.001$ & $<0.001 *$ & $\begin{array}{l}1 \\
\text { (Reference) } \\
4.22(2.77- \\
6.43)\end{array}$ \\
\hline $\begin{array}{l}346 \\
(55) \\
263 \\
(45)\end{array}$ & 0.003 & 0.449 & $\begin{array}{l}1 \\
\text { (Reference) } \\
1.13(0.82- \\
1.57)\end{array}$ & 0.002 & 0.314 & $\begin{array}{l}1 \\
\text { (Reference) } \\
1.23(0.82- \\
1.82)\end{array}$ \\
\hline $\begin{array}{l}489 \\
(78) \\
135 \\
(22) \\
602\end{array}$ & 0.004 & $0.009 *$ & $\begin{array}{l}1 \\
\text { (Reference) } \\
1.66(1.13- \\
2.44)\end{array}$ & 0.005 & $0.037 *$ & $\begin{array}{l}1 \\
\text { (Reference) } \\
1.63(1.03- \\
2.58)\end{array}$ \\
\hline $\begin{array}{l}516 \\
(86) \\
86 \\
(14) \\
625\end{array}$ & 0.015 & 0.269 & $\begin{array}{l}1 \\
\text { (Reference) } \\
1.27(0.83- \\
1.93)\end{array}$ & 0.011 & 0.298 & $\begin{array}{l}1 \\
\text { (Reference) } \\
1.32(0.79- \\
2.20)\end{array}$ \\
\hline $\begin{array}{l}313 \\
(50) \\
312 \\
(50)\end{array}$ & 0.014 & $0.009 *$ & $\begin{array}{l}1 \\
\text { (Reference) } \\
1.54(1.11- \\
2.13)\end{array}$ & 0.039 & $0.046^{*}$ & $\begin{array}{l}1 \\
\text { (Reference) } \\
1.50 \text { (1.01- } \\
2.24)\end{array}$ \\
\hline
\end{tabular}

Table 1. Univariate and multivariate Cox regression analyses of independent predictors of disease-free and overall survival among patients with breast cancer in the NCU cohort. ${ }^{*} P<0.05$ was considered statistically significant. HECTD1 HECT domain E3 ubiquitin protein ligase 1, ER estrogen receptor, $H E R 2$ human epidermal growth factor receptor 2, $H R$ hazard ratio, $C I$ confidence interval.

\section{Figures}




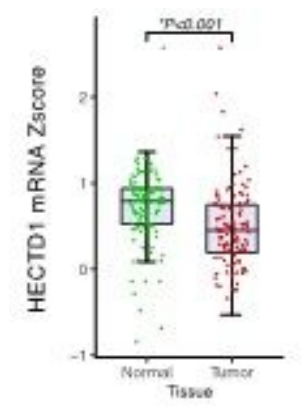

\section{Figure 1}

HECTD1 mRNA expression levels in adjacent normal tissues and breast cancer tissues from the patients in the TCGA cohort. The $Z$ scores of mRNA expression are determined for each sample by comparing the mRNA expression of a gene with its distribution in a reference population showing typical expression of the gene. Vertical lines represent minimum and maximum values; lines within each box represent the median; boxes indicate first to third quartiles; outliers are plotted as individual points. ${ }^{*} \mathrm{P}<0.05$, based on Mann-Whitney test.
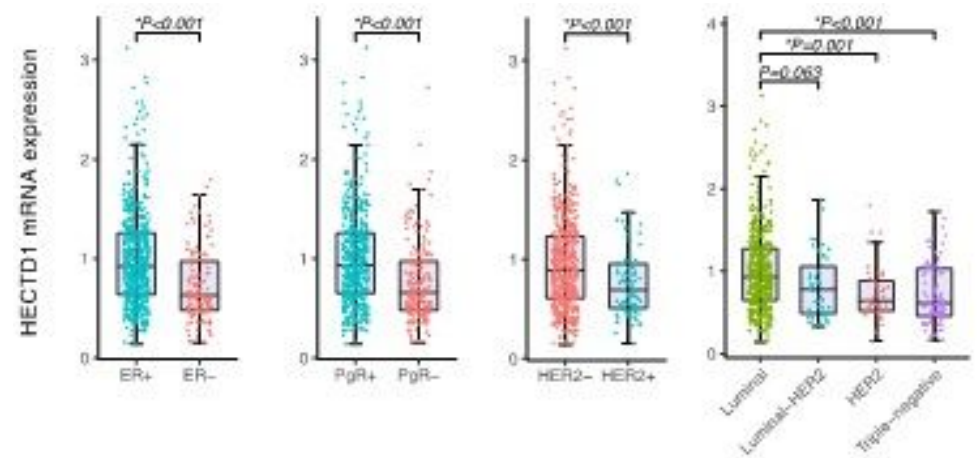

\section{Figure 2}

HECTD1 mRNA expression levels in relation to breast cancer subtype in the NCU cohort. Vertical lines represent minimum and maximum values; lines within each box represent the median; boxes indicate first to third quartiles; outliers are plotted as individual points. ${ }^{*} \mathrm{P}<0.05$, based on Mann-Whitney test.
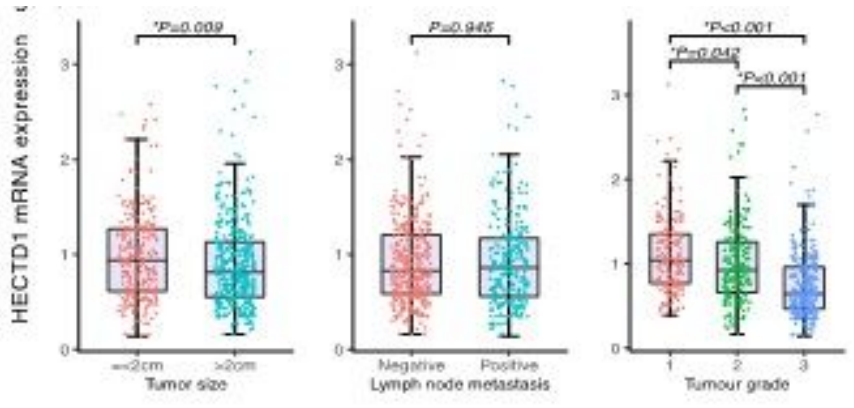

\section{Figure 3}

HECTD1 mRNA expression levels in relation to tumor size, lymph node metastasis, and tumor grade in the NCU cohort. Vertical lines represent minimum and maximum values; lines within each box represent the 
median; boxes indicate first to third quartiles; outliers are plotted as individual points. ${ }^{*} \mathrm{P}<0.05$, based on Mann-Whitney test.

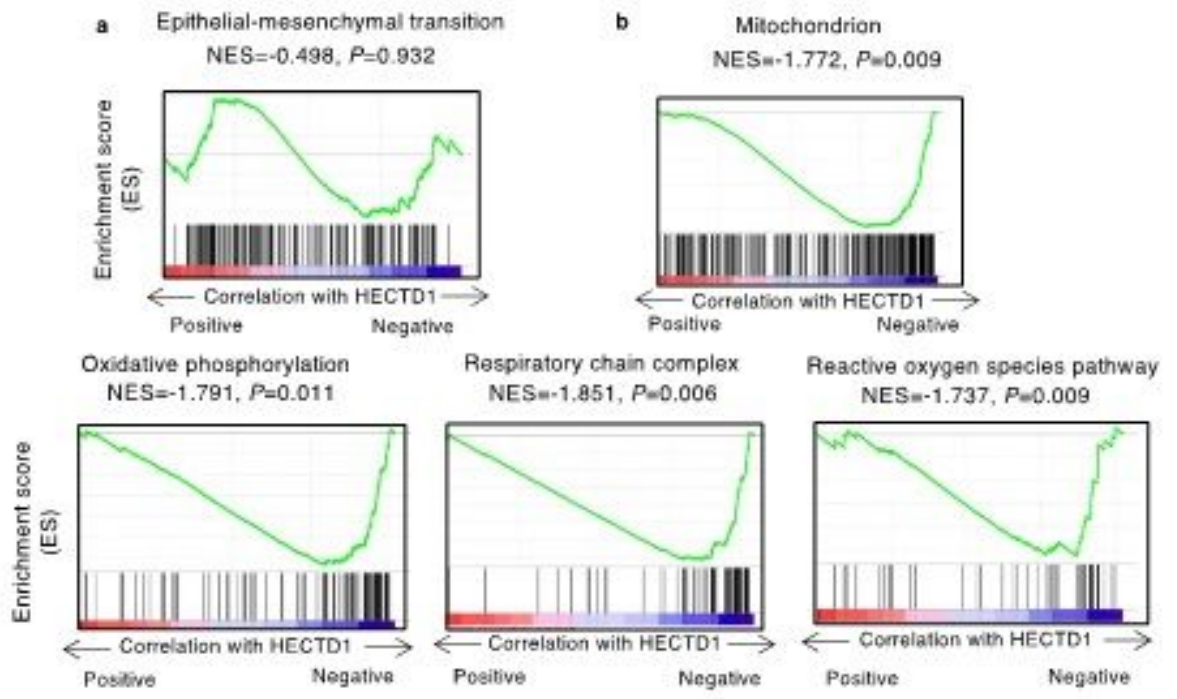

\section{Figure 4}

Correlations between HECDT1 mRNA expression levels and gene set enrichment analysis of (a) epithelialmesenchymal transition and (b) mitochondrion, oxidative phosphorylation, respiratory chain complex, and reactive oxygen species pathway gene sets in the TCGA cohort. NES normalized enrichment score.
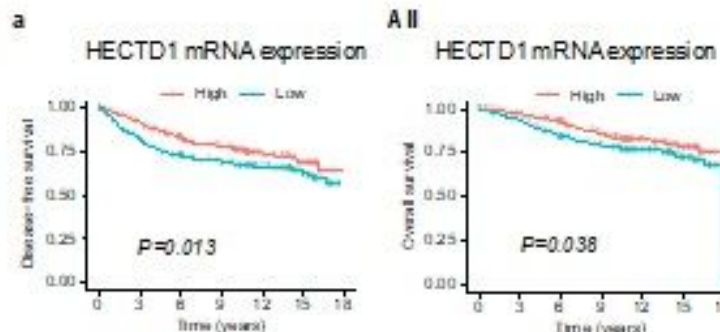

Numbarat nak

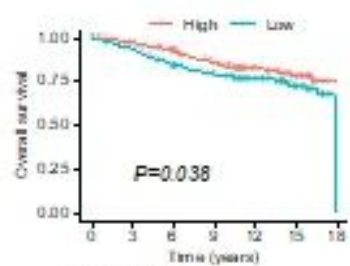

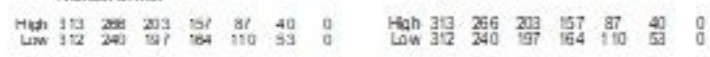

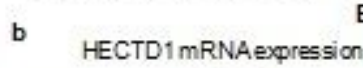

\section{ER-positive}

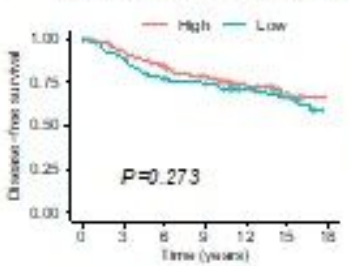

Nuntarainiso.

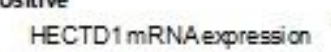

ER-negative

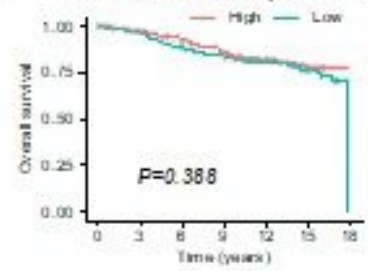

Normas is rote

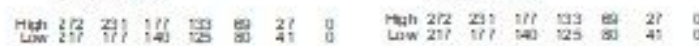

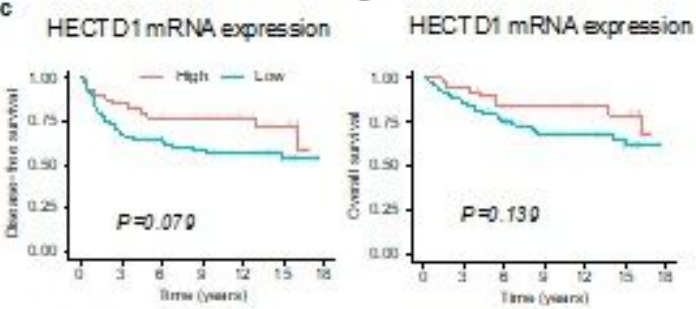

Number serbik

Number is nis

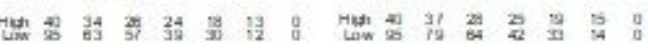

\section{Figure 5}

Kaplan-Meier curves of disease-free survival (DFS) and overall survival (OS) in patients with high and low HECTD1 mRNA expression levels. Graphs show DFS and OS curves, respectively, for (a) all breast cancer patients, (b) ER-positive breast cancer patients, and (c) ER-negative breast cancer patients in the NCU cohort. $\mathrm{P}$ based on log-rank test. 


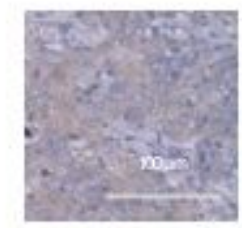

Intensity: 1+

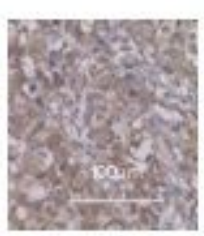

Intensity: 2+

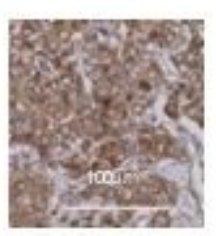

Intersity: 3+
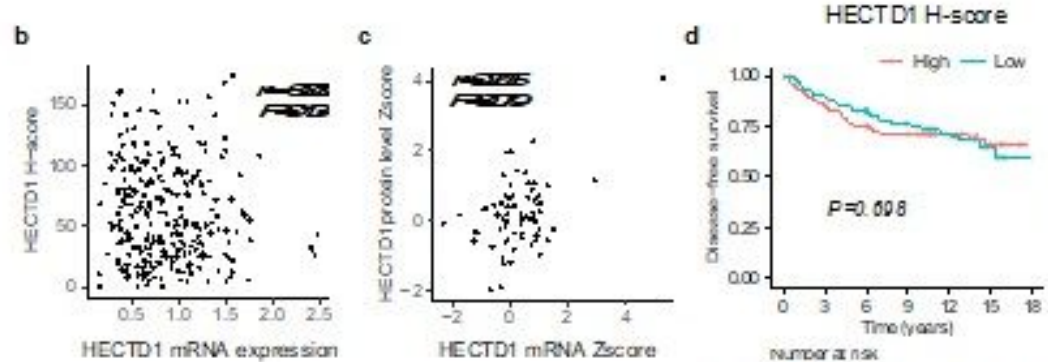

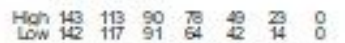

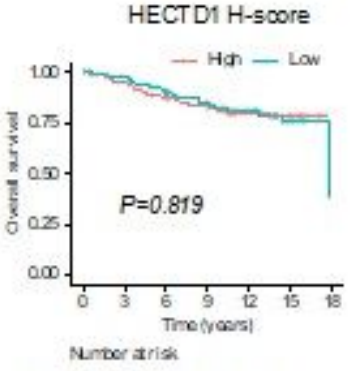

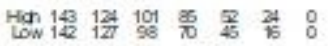

\section{Figure 6}

(a) Representative images of HECTD1 protein expression in breast cancer tissues from the NCU cohort detected by immunohistochemistry. Correlations between HECTD1 mRNA and protein expression levels in the (b) NCU and (c) TCGA cohorts (Spearman's rank correlation coefficient (r)). The Z scores for protein in the TCGA cohort are determined for each sample by comparing with all samples with protein data. (d) Kaplan-Meier curves of disease-free survival (DFS) and overall survival (OS) in patients with high and low HECTD1 protein expression levels. Graphs show DFS and OS curves for all breast cancer patients in the NCU cohort. P based on log-rank test.

\section{Supplementary Files}

This is a list of supplementary files associated with this preprint. Click to download.

- SupplementaryFile.pdf 